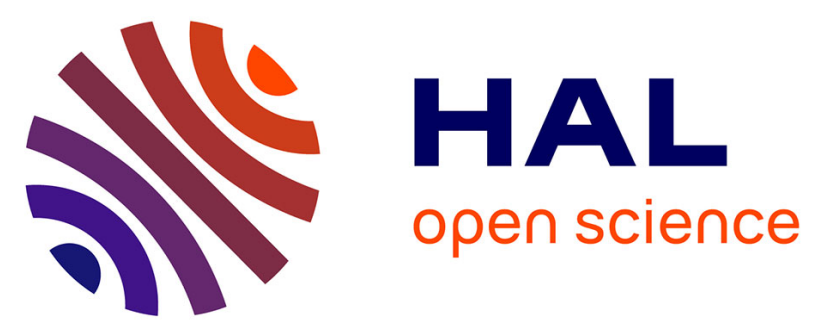

\title{
ESSAIS DE CARACTÉRISATION DE LA SÉGRÉGATION INTERGRANULAIRE DANS LES JOINTS DE GRAINS D'ALLIAGES Ni - Si EN ANALYSE PAR EMISSION X - STEM
}

Luc Beaunier, H. Dexpert, Colette Vignaud

\section{To cite this version:}

Luc Beaunier, H. Dexpert, Colette Vignaud. ESSAIS DE CARACTÉRISATION DE LA SÉGRÉGATION INTERGRANULAIRE DANS LES JOINTS DE GRAINS D'ALLIAGES Ni - Si EN ANALYSE PAR EMISSION X - STEM. Journal de Physique Colloques, 1984, 45 (C2), pp.C2-419-C2-422. 10.1051/jphyscol:1984295 . jpa-00224010

\author{
HAL Id: jpa-00224010 \\ https://hal.science/jpa-00224010
}

Submitted on 1 Jan 1984

HAL is a multi-disciplinary open access archive for the deposit and dissemination of scientific research documents, whether they are published or not. The documents may come from teaching and research institutions in France or abroad, or from public or private research centers.
L'archive ouverte pluridisciplinaire HAL, est destinée au dépôt et à la diffusion de documents scientifiques de niveau recherche, publiés ou non, émanant des établissements d'enseignement et de recherche français ou étrangers, des laboratoires publics ou privés. 


\title{
ESSAIS DE CARACTÉRISATION DE LA SÉGRÉGATION INTERGRANULAIRE DANS LES JOINTS DE GRAINS D'ALLIAGES Ni - Si EN ANALYSE PAR EMISSION $X$ - STEM
}

\author{
L. Beauniert, H. Dexpert ${ }^{*}$ et $C$. Vignaud \\ Groupe de Recherche $n^{\circ} 4$ du CNRS, Physique des Liquides et Electrochimie, \\ Université Pierre et Marie Curie, 4 place Jussieu, 75230 Paris Cedex 05, \\ France \\ * Institut Français du Pétrole, B.P. 311, 92506 Rueit-Malmaison, France
}

Résumé - Nous mettons en évidence la ségrêgation du silicium dans les joints de grains du nickel contenant $1,3 \%$ de silicium atomique en matrice. La mesure est faite par analyse directe des joints de grains en émission $X$ sur STEM.

Abstract - The silicon grain-boundary segregation is shown in a $1.3 \%$ at. nickel-silicon aTToy, by direct measurement on grain-boundaries by spectrometry $X$ on STEM.

Des alliages de nickel contenant diverses teneurs en silicium ont été étudiés par un test de dissolution électrochimique des joints de grains [1]. Les dimensions des fossēs d'attaque intergranulaires sont mesurées, en particulier l'angle d'ouverture $\alpha$. Plus cet angle est étroit plus la profondeur de pénétration est importante. La figure 1 montre l'évolution de ce paramètre pour un traitement de mise en équilibre des joints, $1150^{\circ} \mathrm{C} 4 \mathrm{~h}$, courbe 1 et après traitement de sensibilisation à la corrosion intergranulaire, $1150^{\circ} \mathrm{C} 4 \mathrm{~h}+550^{\circ} \mathrm{C}$ jours, courbe 2 . La corrosion intergranulaire maximum est atteinte pour un taux de silicium proche de $1 \%$ en poids. Un modèle de dissolution [2] permet de calculer les volumes molaires moyens aux joints de grains correspondants -figure2-. La diminution du volume molaire vers $1 \% \mathrm{Si}$ correspondant au maximum de corrosion peut s'expliquer par une sẻgrégation d'un élément lêger tel que le silicium.

Une analyse préliminaire à $1 \mathrm{MeV}$ par pertes d'énergie des électrons avait permis de confirmer la présence de silicium et $n$ 'avait détecté aucun autre élément présent [3].

Les caiculs de Doigt et Flewitt tendent à montrer qu'il serait possible de détecter jusqu'à une monocouche ségrẻgée,en analyse par spectromêtrie dispersive en énergie de rayons $X$ après analyse mathématique du signal [4].

Dans cette étude nous prèsentons les résultats obtenus, pour la teneur 1,3\% si atomique, avec un microscope électronique analytique, à $100 \mathrm{KeV}$, VGHB5 équipé d'un système d'analyse $X$ à dispersion en énergie. Cet appareil permet d'obtenir des faisceaux ponctuels fins jusqu'à $0,5 \mathrm{~nm}$; il est équipé d'une source à émission de champ de forte brillance $\left(10^{10} \mathrm{~A} / \mathrm{cm}^{2} / \mathrm{SR}\right)$. On dispose donc d'une sonde intense qui permet d'obtenir une forte densité de photons $X$ en un point très localisé de l'échantilion.

L'enrichissement en silicium au joint de grain peut être mis en évidence en comparant le signal silicium issu du joint au signal silicium issu de la matrice au voisinage immédiat du joint. Les meilleures conditions géométriques seraient d'aligner le faisceau et le joint de grains. C'est difficilement réarisable compte tenu des instabilités mécaniques et électroniques, de la mauvaise localisation du contraste intergranulaire et de l'amplitude d'inclinaison limitée à $20^{\circ}$ par le porte-objet en graphite. Il est donc nécessaire de travailler avec un plan de joint incliné par rapport au faisceau d'électrons en éliminant le travail en faisceau fixe qui provoque une contamination rapide de la zone analysée. Le cliché $n^{\circ} 1$ présente $]^{\prime}$ 'aspect d'une lame mince avec un joint $A$ parallēle au faisceau, un joint B incliné et trois impacts dus au travail en faisceau fixe. Ceux-ci permettent cependant de mesurer

\footnotetext{
travail effectué dans le cadre d'un détachement CNRS à l'IFP.
} 
T'épaisseur de la lame après inclinaison. Le cliché $n^{\circ} 2$ présente les conditions réelles de travail : joint incliné et balayage en ligne perpendiculaire à la trace du joint.

En faisant abstraction de l'élargissement du faisceau pendant la traversée de la lame nous pouvons définir le rapport du volume total analysé $v$ et du volume réel de joint $V_{J}$

$$
\frac{V}{V_{J}}=\frac{t}{J} \sin \theta \quad \begin{array}{ll}
-t & \text { épaisseur de la lame } \\
& -j \text { epaisseur du joint } \\
& -\theta \text { inclinaison du joint par rapport } \\
\text { au faisceau }
\end{array}
$$

Si on appelle $\beta$ le rapport d'enrichissement intergranulaire on peut calculer le rapport $R$, nombre d'atomes de silicium dans le volume anallysé contenant le joint de grains au nombre d'atomes de Si dans le volume identique pris dans la matrice. Ce rapport reprësente en première approximation le rapport des intensités d'émission $X$ correspondantes.

$$
R=\frac{\beta+\frac{V}{V_{J}}-1}{\frac{V}{V_{J}}}
$$

\begin{tabular}{|c|c|c|c|c|c|c|c|c|c|c|c|c|c|}
\hline : & $\theta$ & 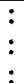 & $\frac{V}{V_{J}}$ & $:$ & $\beta$ & $:$ & $\mathrm{R}$ & $\vdots$ & $:$ & $\Delta N$ & $\begin{array}{l}\vdots \\
\vdots\end{array}$ & C & \\
\hline & & : & & : & 2 & : & 1,5 & : & : & 45 & : & $1,9 \%$ & \\
\hline & $1^{\circ}$ & : & 2,1 & $\vdots$ & 38 & $\vdots$ & 18,70 & 94 & $\vdots$ & 1659 & $:$ & $24 \%$ & \\
\hline : & & : & & : & 2 & : & 1,05 & : & : & 46 & : & 1,36 & $\%$ \\
\hline : & $10^{\circ}$ & $:$ & 20,8 & $:$ & 38 & $:$ & 2,8 & 949 & $:$ & 1684 & $:$ & 3,6 & $\%$ \\
\hline ? & & : & & : & 2 & : & 1,02 & : & : & 48 & : & 1,33 & $\%$ \\
\hline & $20^{\circ}$ & $:$ & 41 & $\begin{array}{l}: \\
:\end{array}$ & 38 & $:$ & 1,90 & $\begin{array}{l}: \\
:\end{array}$ & $:$ & 1765 & $:$ & 2,5 & $\%$ \\
\hline & & : & & : & 2 & : & 1,009 & : & : & 131 & : & 1,31 & $\%$ \\
\hline & $70^{\circ}$ & $:$ & 113 & : & 38 & $:$ & 1,3 & $\begin{array}{l}: 14780 \\
:\end{array}$ & : & 4850 & : & 1,7 & $\%$ \\
\hline
\end{tabular}

On sait que dans ces alliages la ségrégation est localisée sur un très petit nombre de plans atomiques autour du jojnt de grainsaussi fait-on ici l'hypothẽse d'une êpaisseur de joint $J$ égale à $5 \AA$ et en prenant pour moyenne une épaisseur de lame $t$ de $600 \AA$ on peut établir le tableau 1 .

TABLEAU 1

Dans le tableaú 1, nous présentons, pour deux valeurs d'enrichissement $\beta$ et divers angles $\theta$, les valeurs du rapport $\frac{V}{V_{J}}$, du rapport $R$, du nombre d'atomes de silicium

analysés dans la matrice seule $N$ et du supplēment d'atomes de $S i$ à dētecter $\Delta N$ quand on analyse le même volume contenant le joint de grains. On en déduit aussi la teneur apparente $C$ en silicium dans ce même volume.

Dans nos conditions de travail (inclinaison des joints de grains non corrigeables) nous avons enregistrés des spectres qui pouvaient correspondre à des rapports $R$ quelconques dans la gamme de 1,009 à 1,5 pour $\beta=2$ et de 1,3 à 18,7 pour $\beta=38$ (50\% Si au joint de grains). 
Malgré des analyses de $200 \mathrm{~s}$ à $500 \mathrm{~s}$ la statistique de comptage est faible et l'erreur relative importante; par ajlleurs l'épaisseur de la lame est suffisante pour que $T^{\prime}$ 'on ait un continuum dont la soustraction nécessite un modèle. D'autre part, on note que pour $B=2$, avec la meilleure géométrie donc le plus grand rapport $R=1,48$, le nombre d'atomes de $S i$ supplémentaires est trop faible ; en effet le nombre minimum d'atomes étrangers détectables dépend fortement du nombre totald'atomes analysés.

Dans l'hypothèse d'une valeur de $\beta$ voisine de 2 la concentration apparente en Si à détecter varie entre $1,31 \%$ atomique et $1,9 \%$ atomique alors que la concentration nominale dans la matrice $C_{o}$ est de $1,3 \%$ atomique. La situation est beaucoup plus favorable pour $B=38$ : même pour des joints inclinés la teneur apparente dépasse $2 \%$.

La figure $n^{\circ} 3$ correspond à un cas où $R=1,25$, ce qui pour ce joint incliné de $70^{\circ}$ correspondrait à un facteur $\beta$ voisin de 30 ; la majorité des joints se trouvent dans un cas souvent plus défavorable, $i 1$ arrive même d'observer des intensités du pic Si plus faibles sur certains joints que sur la matrice voisine, peut-être s'agit-il de zones appauvries au voisinage du joint.

Dans certaines lames les spectres individuels ne montrent pas l'effet de ségrégation. Nous avons été amenés à sommer les intensités des spectres de $n$ joints de grains et des n matrices correspondantes. La figure 4 présente ce résultat qui met en évidence le phênomène moyen de ségrêgation en améliorant le bruit statistique donc le rapport pic sur fond continu. Dans ce cas la valeur $R$ mesurêe est une moyenne des différentes géométries d'analyse.

Au cours de nos analyses nous avons souvent enregistré la présence d'un pic de soufre qui pouvait être plus intense pour le spectre-joint que pour le spectre-matrice correspondant. Ceci confirme notre hypothèse d'une coségrégation soufre-silicium aux joints de grains dans l'alliage étudiē [5]. Alors que le soufre ségrégé est un élément fragilisant, le silicium, même à des teneurs élevées, ne permet pas d'obtenir des fractures totalement intergranulaires. Cependant de récents essais nous laissent espërer qu'il sera bientôt possible d'aborder tant aux joints de grains qu'en surface, ce phénomène complexe de compétition de ségrégation, des élēments soufre et silicium dans le nickel par spectromètrie des électrons Auger.

\section{Rêférences}

[1] BEAUNIER L., FROMENT M., C.R. Acad. Sci. tome 279 (1974) 91C.

[2] BEAUNIER L., FROMENT M., VIGNAUD C., Electrochimica Acta, 25 (1980) 1239.

[3] BEAUNIER L., FROMENT M., VIGNAUD C., J. Microsc. Spectrosc. Electron., vol. 3 (1978) p. 265.

[4] DOIGT P., FLEWITT P.E.J., Inst. Phys, Conf. Ser., 61 (1981) P 167 EMAG Cambridge 1981.

[5] BEAUNIER L., FROMENT M., VIGNAUD C., 164Th Meeting of the Electrochemical Sty., 9 Oct. 1983, Washington DC (USA). Proceedings of the Symposium : "Fundamental aspects of corrosion, protection by surface modification". 

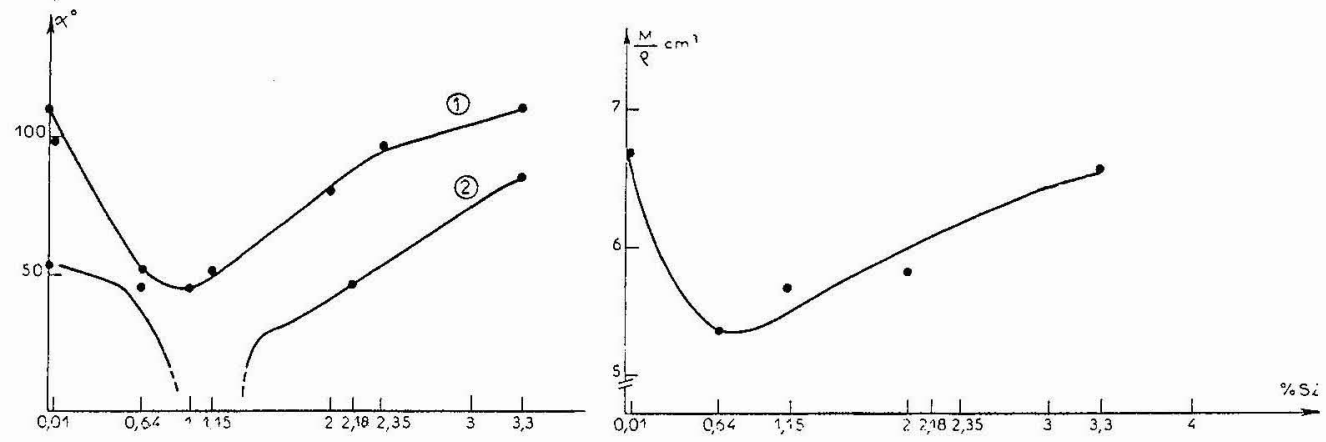

Fig. 1 - angle $\alpha$ du sillon intergranulaire $1-1050^{\circ} \mathrm{C} 4 \mathrm{~h}$

$2-1050^{\circ} \mathrm{C} 4 \mathrm{~h}+550^{\circ} \mathrm{C}$ jours

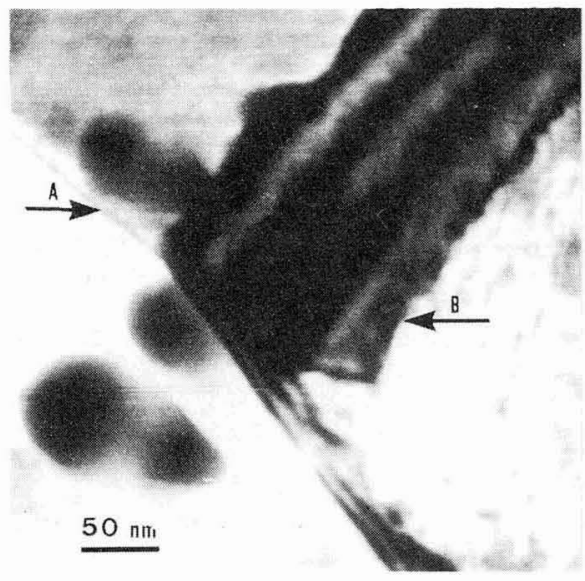

Fig. 2 - M/o Volume molaire moyen aux joints de grains

$-1050^{\circ} \mathrm{C} 4 \mathrm{~h}$

CLI. 1. - impacts

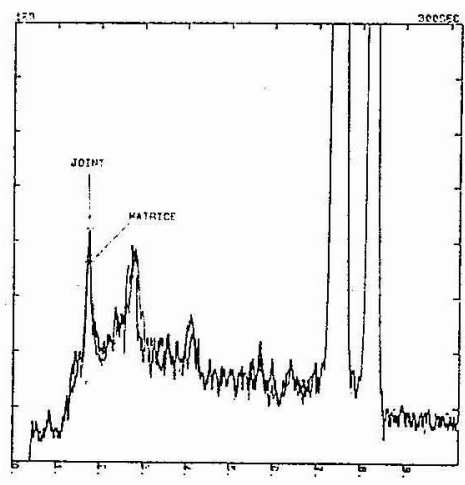

D. onver

Fig. 3 - Spectres obtenus

- en traversée perpendiculaire aux joints

- sur la matrice seule

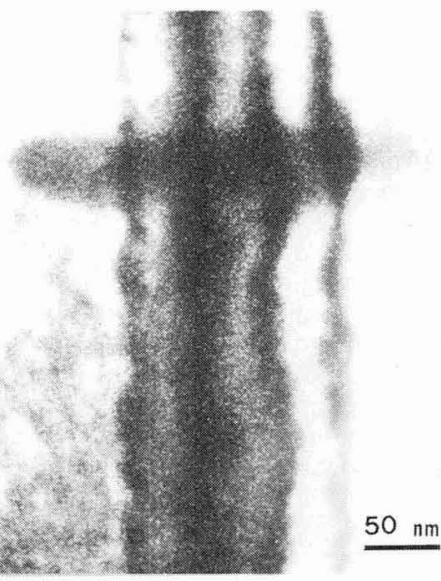

CLI. 2 - Traversée

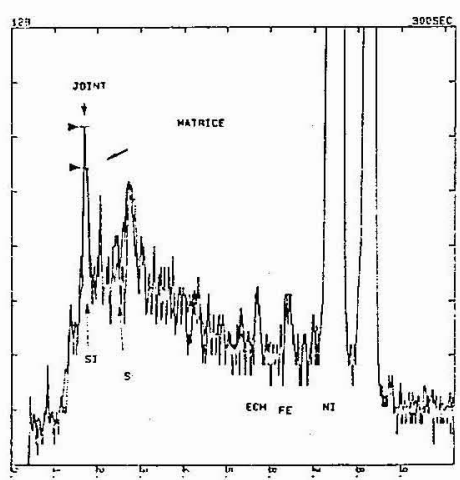

p.estex

Fig. 4 - Somme de

- $n$ spectres-"joint"

- $n$ spectres - "matrice" 